\title{
TRABALHO EM EQUIPE: UMA REVISITA AO CONCEITO E A SEUS DESDOBRAMENTOS NO TRABALHO INTERPROFISSIONAL
}

\author{
TEAMWORK: REVISITING THE CONCEPT AND ITS \\ DEVELOPMENTS IN INTER-PROFESSIONAL WORK
}

\begin{abstract}
Marina Peduzzi ${ }^{1}$ (D) (0000-0002-2797-0918), Heloise Lima Fernandes Agreli² ${ }^{2}$ (D) (0000-0002-7234-836X), Jaqueline Alcântara Marcelino da Silva ${ }^{3}$ (iD) (0000-0002-8307-8609), Helton Saragor de Souza ${ }^{4}$ (ID) (0000-0002-3074-6386)
\end{abstract}

\author{
${ }^{1}$ Universidade de São Paulo, Escola de Enfermagem, Departamento de Orientação Profissional, São Paulo, \\ SP, Brasil. <marinape@usp.br> \\ ${ }^{2}$ Universidade de São Paulo, Escola de Enfermagem, Departamento Orientação Profissional, São Paulo, \\ SP, Brasil; e Emlyon École de Management, Lyon, França. \\ ${ }^{3}$ Universidade Federal de São Carlos, Departamento de Enfermagem, São Carlos, SP, Brasil. \\ ${ }^{4}$ Universidade Federal de São Paulo, Campus Baixada Santista, Departamento de Políticas Públicas e \\ Saúde Coletiva. Santos, Brasil.
}

Resumo A crescente complexidade das necessidades de saúde que requerem abordagem ampliada e contextualizada e da organização dos serviços em rede motivou a revisita ao conceito de trabalho em equipe, à tipologia equipe-integração e agrupamento e aos seus atributos. O efetivo trabalho em equipe se constitui como expressão, por um lado, da articulação das ações das diversas áreas mediante o reconhecimento da sua interdependência e, por outro, da complementaridade indispensável entre agir instrumental e agir comunicativo. Destaca-se que o trabalho em equipe também é expressão da divisão social do trabalho e da transformação de diferenças técnicas em desigual valor social dos trabalhos especializados das diversas profissões e, portanto, dos seus agentes. As contradições presentes nos processos de trabalho, na sua consubstancialidade às necessidades de saúde, podem se limitar a reiterar os modelos dominantes de atenção à saúde ou buscar sua superação por meio de mediações reflexivas e dialógicas dos trabalhadores das equipes entre si e destes com usuários, famílias e população. Por fim, trabalho em equipe constitui uma das formas de trabalho interprofissional com práticas colaborativas, e essa colaboração pode se dar na equipe ou no trabalho em rede com usuários e comunidade.

Palavras-chave equipe de assistência ao paciente; relações interprofissionais; trabalho.
Abstract The increasing complexity of health needs that require expanded and contextualized approach and the organization of networked services has motivated revisiting the concept of teamwork, the team-integration and grouping typology and its attributes. Effective teamwork is an expression, on the one hand, of the articulation of the actions of the various areas from the recognition of their interdependence and, on the other, of the indispensable complementarity between instrumental and communicative action. It is noteworthy that teamwork is also an expression of the social division of labor and the transmutation of technical differences into unequal social value of specialized work of different professions and, therefore, of their agents. The contradictions present in work processes, in their substantiation with health needs, may be limited to reiterating the dominant models of health care or seeking to overcome them through reflective and dialogical mediations of team workers among themselves and with users, families and population. Finally, teamwork is one of the forms of interprofessional work together with interprofessional collaboration and collaborative practice, and this collaboration can take place in the team or in networking with users and the community. Keywords patient care team; interprofessional relations; work. 


\section{Introdução}

A proposta de trabalho em equipe vem sendo discutida e implementada desde os anos 1950 (Peduzzi, 2009) e recebe novo destaque por parte da Organização Mundial da Saúde e outros organismos nacionais e globais a partir dos anos 2000, culminando com a publicação de um marco de referência para prática e educação interprofissional em 2010 (Organização Mundial da Saúde-OMS, 2010).

A imprecisão dos conceitos e dos termos utilizados expõe que ainda não foi possível construir um consenso, mesmo que provisório, sobre os elementos-chave que constituem o trabalho em equipe e suas variações: multidisciplinar, interdisciplinar, transdisciplinar ou multiprofissional e interprofissional.

De maneira geral, os prefixos multi, inter e trans, nesta sequência, denotam um grau crescente de interação, integração e coordenação das disciplinas ou profissões segundo o termo utilizado a seguir, disciplinar ou profissional, que fazem referência, respectivamente, ao âmbito das áreas de conhecimento ou disciplinas e das práticas profissionais (Furtado, 2007; Peduzzi et al., 2013).

Há uma vasta literatura internacional sobre prática e educação interprofissional no campo da saúde que expressa necessidade de se avançar das equipes multiprofissionais para as equipes interprofissionais. Este artigo constitui um ensaio que trata das concepções de trabalho em equipe interprofissional e de outros dois construtos correlatos - colaboração interprofissional e prática colaborativa interprofissional (D'Amour et al., 2008; Morgan, Pullon e McKinlay, 2015; Reeves et al. 2010; Reeves, Xyrichis e Zwarenstein, 2018). Também trata do construto clima de equipe, visto que suas quatro dimensões permitem uma aproximação mais operacional do trabalho em equipe nos serviços de saúde configurando ferramenta da gestão do trabalho (West e Richter, 2011).

O destaque para o tema nas duas últimas décadas está relacionado ao reconhecimento de que trabalho em equipe e prática colaborativa interprofissional contribuem para melhorar o acesso e a qualidade da atenção à saúde (Zwarenstein, Goldman e Reeves, 2009; OMS, 2010; Reeves et al., 2016, 2017). A literatura também aponta seu potencial para melhorar a experiência de paciente e familiares no cuidado à saúde e o custo-efetividade da atenção à saúde (Brandt et al., 2014), bem como a experiência no trabalho cotidiano dos profissionais de saúde (Song et al., 2017; Chang et al., 2009; Chiang Vega et al., 2011). Com isso, observa-se que o trabalho em equipe tem potencial e pode, por um lado, produzir melhores resultados na atenção à saúde de usuários, família e comunidade e, por outro, melhorar a satisfação no trabalho por parte dos profissionais/trabalhadores.

O trabalho em equipe é necessário e constitui um dos componentes estratégicos de enfrentamento da crescente complexidade, tanto das necessidades de saúde que requerem uma abordagem ampliada e contextualizada como da 
organização dos serviços e dos sistemas de atenção à saúde em rede. Também decorre da mudança demográfica com aumento da expectativa de vida e envelhecimento da população, assim como da mudança do perfil epidemiológico com crescimento das doenças e condições crônicas, que requerem acompanhamento de parte da população por anos e décadas, o que resulta melhor se os serviços de saúde se organizam com base em equipes.

A crescente tendência de trabalho interprofissional motivou a revisita ao conceito de trabalho em equipe, sua tipologia e seus atributos, apresentados por Peduzzi (2001) com base no mesmo referencial teórico - processo de trabalho em saúde e teoria do agir comunicativo, agora em diálogo com a literatura internacional sobre o tema do trabalho em equipe publicada nas duas últimas décadas. Trata-se o trabalho em equipe como trabalho e processo de trabalho, visto que equipes de saúde dizem respeito à execução de ações orientadas a um fim (Habermas, 2009). Não se diz equipes de pacientes, equipes de familiares; poderia se dizer grupos de pacientes, grupos de familiares. As equipes de saúde, no entanto, são equipes de trabalho.

Inicialmente o texto contextualiza a análise no campo da saúde coletiva, em particular com base nas contribuições da categoria processo de trabalho de Ricardo Bruno Mendes-Gonçalves (Mendes-Gonçalves, 1992, 1994; 2017) e suas interfaces com a categoria trabalho do campo das ciências sociais. Isso remete a uma abordagem do trabalho em equipe que leva em consideração as contradições presentes na divisão social do trabalho e sua contínua reprodução das desigualdades sociais.

Ricardo Bruno foi discípulo de Cecília Donnangelo e parte da análise presente em Donnangelo e Pereira (1976) acerca da medicina consubstancialmente como prática técnica e social para fundamentar a investigação do caráter social do trabalho médico. Na sua análise histórica e social, dá destaque ao processo de trabalho e seus componentes - atividade, objeto de trabalho, instrumentos (saberes, tecnologias e organização tecnológica do trabalho), finalidade e agentes - e às necessidades de saúde que, na sua relação dialética processo de trabalho e necessidades, permite compreender as práticas de saúde na sua articulação com as demais práticas sociais. Cabe destacar, como alerta o autor, que mesmo "partindo da convicção de que a dimensão-trabalho das práticas sociais constitui-se na base mais fundamental de sua efetivação, elas não se reduzem a essa dimensão, e portanto não devem ser reduzidas a essa dimensão pelo pensamento que queira explicá-las". O autor destaca que toda teoria deve ser mantida em constante e severa vigilância, "pois as teorias não são propriamente adequadas em si mesmas, mas tornam-se adequadas à medida que as práticas se tornam adequadas e impõem sua retificação" (Mendes-Gonçalves, 2017, p. 300-301, destaques no original).

A literatura sobre trabalho em equipe também evidencia a comunicação e interação social como elemento sine qua non, o que levou a adoção da teoria 
do agir comunicativo de Jürgen Habermas como referencial articulado ao de processo de trabalho em saúde. Com base no quadro teórico apresentado a seguir, o artigo segue dialogando com a literatura sobre trabalho em equipe, colaboração interprofissional, prática colaborativa e clima de equipe.

\section{Processo de trabalho interprofissional: agir instrumental e agir comunicativo}

A análise acerca da atividade dos profissionais de saúde está presente nos estudos fundacionais da saúde coletiva. Tanto Donnangelo e Pereira (1976) quanto Arouca (2003) interpretam a prática médica na estrutura social capitalista e fundamentam a teoria social da medicina e da saúde. Os seus objetos centrais de pesquisa remetem ao significado da ampliação da clínica, à generalização do consumo e à racionalização dos serviços, sendo uma expressão dessa relação sócio-histórica a medicina comunitária nos primeiros autores citados, e a crítica à ideologia e à formação discursiva da medicina preventiva no segundo autor.

As práticas de saúde somente poderiam ser entendidas na articulação com as demais práticas sociais, especificamente no papel de reconstituição das condições necessárias para a reprodução da força de trabalho (corpo dos sujeitos como agente de trabalho) e atenuação das tensões sociais próprias da desigualdade na sociedade capitalista. Com referência à atividade laboral propriamente dita, os autores se concentraram na principal figura do processo contemporâneo da medicalização e da generalização da clínica pelo Estado (Foucault, 2002), o médico.

Donnangelo e Pereira (1976), por exemplo, assinalam que a atividade do médico deveria ser entendida como trabalho e não como mero exercício técnico científico neutro e que o objeto da medicina, a doença instalada no corpo, decorre das transformações sobre o corpo ou meio físico socialmente determinadas, e não somente conforme a prescrição biomédica de redução anatomofisiológica. Arouca (2003) define o cuidado como o processo de trabalho de natureza relacional entre o médico e o paciente, no qual o médico é intermediário da relação de troca de mercadorias da indústria. Esses autores já diagnosticaram, nos anos 1970, a crescente incorporação tecnológica e de capital que complexifica o trabalho em saúde e solapa majoritariamente o exercício liberal do profissional médico.

Mendes-Gonçalves (2017) mantém a abordagem marxiana, ${ }^{1}$ mas introduz uma mudança no âmbito teórico e de pesquisa, visto que coloca o entendimento das práticas em saúde na sua articulação social, sobretudo nos processos de trabalho. No plano empírico, aborda as diversas ocupações em saúde pioneiramente (Mendes-Gonçalves, 1992; 1994). O autor destrincha a divisão social capitalista em saúde que se sobrepõe à dimensão técnica e expõe que o conjunto das práticas em saúde é realizado pela figura abstrata do trabalho coletivo. A cooperação entendida a partir do pressuposto teórico da totalidade 
do trabalho humano associado com uma finalidade estabelecida se expressa na atividade do conjunto das categorias profissionais em saúde contemporaneamente. O autor não ignora a existência de hierarquias e desigualdades entre as profissões; contudo, analisa e supera essas relações compreendendo-as como novas formas de processos de trabalho. Desse modo, Mendes-Gonçalves (2017), sem prescrever modelos 'ideais', aposta em novos processos de trabalho conectados com as necessidades radicais em saúde e o propósito de mudança social. As combinações desses processos têm a potencialidade de corresponder às necessidades em saúde conformadas socio-historicamente por meio da práxis do trabalho coletivo. O campo da saúde coletiva, por meio de diversas leituras teóricas, constitui debates e possíveis inflexões nos processos de trabalho, que conformariam novos modelos de atenção orientados às necessidades de saúde da população brasileira, sem reiterar o modelo tradicional biomédico e fragmentado.

A análise do trabalho em equipe multiprofissional (Peduzzi, 2001) fundamenta-se em parte no legado teórico da saúde coletiva aqui referido e considera o crescente aumento da complexidade do objeto peculiar do trabalho em saúde: as necessidades manifestas pelos usuários e população demandante de entendimento dos profissionais de saúde. Nesse sentido, propõe outros parâmetros para a interação entre os agentes e os domínios técnicos específicos providos em parte pela ciência, que devem se articular na constituição de acordos por meio da reciprocidade da fala, alteridade e reconhecimento intersubjetivo (Habermas, 2012). A articulação dos processos de trabalho das diversas áreas constitutivas do trabalho em saúde, operada na experiência do cotidiano de trabalho dos serviços, permitiria forjar outra concepção de cuidado e de atenção à saúde com o horizonte normativo da saúde como valor e direito.

Nesse sentido, a dimensão reflexiva intersubjetiva e a dimensão geral do sujeito exigem articulação de conhecimentos e saberes, pois persistem as tensões entre o exercício da autonomia técnica e o diálogo cotidiano na definição da efetivação dos modelos de saúde. A constituição de equipes interdisciplinares e interprofissionais não é um modelo de resolução definitiva do conjunto dessas tensões, mas é uma perspectiva que tem como pressupostos: a superação da fragmentação do trabalho e da individualização biomédica; a busca de reconstituição da integralidade do trabalho coletivo em saúde; e a qualificação do conjunto dos profissionais sob esses signos que visa democratizar o contexto do trabalho e efetivar integralmente o cuidado.

A teoria do agir comunicativo, elaborada por Jürgen Habermas em 1981 (Habermas, 2012), pode contribuir para a compreensão da interação no trabalho em equipes interprofissionais de saúde. Schraiber e colaboradores (1999), Peduzzi (2001; 2007), Rivera e Artmann (1999), Ayres (2001), Artmann (2001) e Rivera (2003) são pesquisadores do campo da saúde coletiva que incorporaram a análise do processo de trabalho em saúde à luz da teoria do agir comunicativo. 
Habermas desenvolveu uma teoria crítica da sociedade relacionada à construção da democracia por meio da razão, das práticas políticas, sociais e comunicativas. Pautado em Hegel, define a linguagem como expressão do pensamento de um povo e dos valores sociais compartilhados (Habermas, 2009). A partir do pressuposto de um horizonte ético, normas e convicções comuns, a linguagem constitui a ação comunicativa que pode ser compreendida por meio de duas lógicas, monológica ou dialógica, também chamadas de racionalidades instrumental e comunicativa. O agir estratégico e instrumental faz parte da primeira, enquanto na segunda está o agir comunicativo, que pressupõe o diálogo e o entendimento dos sujeitos envolvidos (Habermas, 2009).

$\mathrm{Na}$ interação intersubjetiva, o entendimento entre os sujeitos apoia-se em pretensões de validade: a verdade proposicional que se refere à realidade do estado das coisas, a correção normativa que remete aos aspectos éticos, políticos e morais que coordenam as ações, e a autenticidade expressiva que envolve uma relação de confiança e sinceridade entre os interlocutores (Habermas, 2012). Assim, na relação profissional-usuário, a verdade proposicional pode ser aplicada ao se realizar uma consulta e comunicar os resultados de exames clínicos; a correção normativa, quando se realizam recomendações de autocuidado para promoção ou recuperação da saúde; e a autenticidade expressiva permeia toda interação exemplificada, por meio do vínculo intersubjetivo que requer a escuta, o afeto, a confiança e a dedicação de tempo que possibilitam o acompanhamento longitudinal do cuidado.

Ao aplicar os conceitos habermasianos, vale destacar que os conflitos permeiam as relações intersubjetivas cotidianas nos serviços de saúde. Nesse sentido, espera-se que os profissionais adotem atitudes reflexivas argumentativas, em busca de restaurar o consenso e a vontade comum, mediante a cooperação, com foco nas necessidades dos usuários, famílias e comunidades, considerando a construção de acordos interprofissionais e do espaço de protagonismo do usuário nas relações de cuidado. A ausência da voz do usuário, em virtude da relação tradicionalmente assimétrica estabelecida pelos profissionais, é usual nos serviços de saúde, mas convive com a construção de oportunidades de expressão e valorização dos posicionamentos dos usuários por parte dos profissionais de saúde, para gradativamente consolidar sua participação protagonista no cuidado.

Habermas considera que há uma relação recíproca entre trabalho e interação, com base no argumento de que as regras técnicas que permeiam o trabalho são necessárias para o agir instrumental, e este decorre do agir comunicativo (Habermas, 2009). Assim, pode-se dizer que o produto do processo de trabalho em saúde será um resultado da articulação indispensável entre o agir comunicativo e o instrumental, no qual ocorre a mediação reflexiva e dialógica dos trabalhadores da equipe interprofissional com os usuários, famílias e comunidade em busca do entendimento para a aplicação pertinente 
e contextualizada dos saberes técnico-científicos, assim como de um conjunto maior de saberes e experiências pertinentes à atenção às necessidades de saúde.

Pode-se dizer que, no processo de trabalho em saúde, a aplicação do conhecimento técnico-científico de cada profissional constitui o agir instrumental. E o agir comunicativo envolve a busca do entendimento entre os trabalhadores da equipe de saúde e destes com os usuários e famílias, tanto na tomada de decisão como na construção de objetivos comuns para o cuidado (Peduzzi, 2001). O processo de entendimento entre os sujeitos envolvidos se estabelece com base no mundo da vida que envolve a cultura, a sociedade e a personalidade (Habermas, 2003a, 2012). No cotidiano dos serviços de saúde, espera-se que profissionais de diferentes áreas e usuários compartilhem elementos do mundo da vida e com isso possam forjar e alavancar a construção conjunta da integralidade do cuidado.

O mundo da vida pautado em valores intersubjetivamente compartilhados entre os profissionais de saúde e os usuários pressupõe o investimento na aproximação da linguagem entre os sujeitos, para que ocorra sua efetiva participação na tomada de decisão sobre as necessidades de saúde e seus enfrentamentos, por meio da interação intersubjetiva que possibilita compreensão da complexidade do cuidado. Contudo, o mundo da vida pode ser colonizado com o avanço da racionalidade técnico-científica em esferas da atenção à saúde que requerem prática comunicativa, prejudicando a construção de acordos mútuos entre profissionais e destes com os usuários e famílias.

O espaço do entendimento intersubjetivo pode ser ampliado à esfera pública, cuja definição remete à organização social grega da 'pólis' compartilhada pelos cidadãos livres (Habermas, 2003b). Os profissionais e usuários do Sistema Único de Saúde (SUS) ocupam a esfera pública na medida em que exercem o controle social em espaços democráticos, como os conselhos gestores locais, Conselhos Municipais de Saúde e Conselho Nacional de Saúde, ou quando se engajam em outros movimentos que se relacionam com a representação de interesses coletivos, na busca de direitos sociais e melhorias no acesso à saúde, previstos constitucionalmente. As reuniões de equipe e outros espaços de troca formais ou informais também podem ser considerados no âmbito da esfera pública, já que visam à construção de acordos interprofissionais que representam a cogestão dos compromissos políticos sociais assumidos no âmbito do processo de trabalho dos serviços de saúde.

\section{0 trabalho em equipe e as diferentes formas de trabalho interprofissional}

Ao revisitar o conceito de trabalho em equipe multiprofissional, observa-se que a analise anterior já buscava fundamentar a necessidade de uma interação e integração mais abrangente e horizontal entre os profissionais das diferentes áreas da saúde (Peduzzi, 2001), à medida que concebe trabalho em equipe como trabalho coletivo que se configura na relação recíproca en- 
tre as intervenções técnicas e as interações dos múltiplos agentes envolvidos. A concepção se apoia no entendimento da reciprocidade, ou seja, da lógica dialética, que fundamenta as relações entre ação instrumental e ação comunicativa intersubjetiva. Quanto mais dialógicas forem as relações de trabalho, mais integradas serão as ações de cuidado à saúde; quanto menos dialógicas as relações de trabalho, mais fragmentada resultará a dinâmica das equipes de atenção à saúde.

Ao apresentar a concepção de trabalho em equipe aqui referida, Peduzzi (2001) também traz uma tipologia: equipe-integração e equipe-agrupamento e os atributos que permitem identificar cada um dos tipos - articulação das ações, comunicação entre os profissionais, construção de um projeto comum, divisão social do trabalho que transmuta diferenças técnicas em desigual valor social dos agentes, reconhecimento das especificidades dos trabalhos especializados, autonomia profissional de caráter interdependente, flexibilidade da divisão do trabalho e interdependência das ações e profissões.

A literatura internacional sobre o tema também apresenta tipologias e atributos ou características do trabalho em equipe que permitem diferenciar as modalidades de equipes (D'Amour et al., 2008; Hollenbeck, Beersman e Schouten, 2012; West e Lyubovnikova, 2013). Os autores se valem de referenciais teóricos distintos: D'Amour e colaboradores (2008) utilizam o referencial da ação coletiva do campo da sociologia organizacional com base em Michel Crozier e Erhard Friedberg; Hollenbeck, Beersman e Schouten (2012), assim como West e Lyubovnikova (2013), fundamentam suas tipologias no modelo do campo da gestão denominado input-process-output (IPO).

D'Amour e colaboradores (2008) constroem três tipos de colaboração em equipe: colaboração ativa, colaboração em desenvolvimento e colaboração latente. West e Lyubovnikova (2013) definem dois tipos: equipes reais e pseudoequipes; estas são as apenas denominadas de equipe, mas não apresentam suas características principais. Hollenbeck, Beersman e Schouten (2012) fazem uma crítica às tipologias e propõem a busca de um consenso acerca das dimensões cruciais que caracterizem o trabalho em equipe e sejam úteis para diferenciar as equipes mais e menos efetivas, ou seja: diferenciar as habilidades/especificidades de cada área profissional, diferenciar as autoridades para tomada de decisão e estabilidade temporal.

A literatura apresenta outra abordagem que faz a crítica das tipologias, apontando, sobretudo, duas limitações: a primeira referida à tendência das tipologias de colocar as equipes num espectro de 'pobres equipes' (que não trabalham de forma integrada e com interações frequentes) a 'boas equipes' (que compartilham uma abordagem integrada e interagem regularmente). A segunda crítica se refere à abordagem normativa e linear que as tipologias de trabalho em equipe sugerem (Reeves et al., 2010). Para esses autores, o trabalho em equipe interprofissional é mais complexo do que as tipologias sugerem e 
varia segundo seus atributos, ou seja, mesmo identificadas suas características centrais, estas se apresentam de forma distinta nas variadas equipes. Com base nessa análise, Reeves e colaboradores (2010) propõem uma abordagem contingencial com base em dois aspectos fundamentais: a finalidade ou propósito do trabalho em equipe que está relacionado às necessidades locais de usuários, famílias e comunidade; e as características e condições de trabalho concretas nas quais atuam os profissionais.

Com base na abordagem contingencial, têm sido descritas distintas modalidades de trabalho interprofissional, como: trabalho em equipe, colaboração, prática colaborativa e trabalho em rede (Reeves et al., 2010; Xyrichis, Reeves e Zwarenstein, 2018; Reeves, Xyrichis e Zwarenstein, 2018).

Reeves e colaboradores (2010) também criticam o foco no trabalho em equipe como principal forma de trabalho interprofissional. Segundo os autores, o cotidiano do cuidado em saúde demanda que as equipes se alternem entre as diferentes modalidades de trabalho interprofissional, que pode ser trabalho em equipe ou colaboração e prática colaborativa para além da equipe, ou ainda trabalho em rede.

Ao mesmo tempo que a abordagem contingencial do trabalho interprofissional critica o modelo linear descrito nas tipologias do trabalho interprofissional, ela dialoga com os princípios descritos por tais tipologias. A abordagem contingencial utiliza como forma de diferenciação entre as modalidades do trabalho interprofissional os mesmos dois princípios descritos por Peduzzi (2001) na diferenciação entre equipes-integração e equipe-agrupamento: grau de articulação entre as ações e de interação dos agentes. Os dois princípios são também descritos na tipologia de D'Amour e colaboradores (2008), a qual considera a conectividade e o compartilhamento como princípios para diferenciação entre níveis de colaboração. A aproximação teórica entre Reeves e colaboradores (2010), Peduzzi (2001) e D'Amour e colaboradores (2008) sugere consenso entre os autores acerca do papel da articulação das ações e da interação dos agentes como aspectos centrais para diferenciação entre modalidades do trabalho coletivo interprofissional.

De acordo com Reeves e colaboradores (2010), para diferenciar as formas de trabalho interprofissional é preciso considerar a articulação das ações, que requer o reconhecimento da sua interdependência e a interação dos sujeitos envolvidos, expressa pelo nível de compartilhamento de valores, objetivos e identidade entre os profissionais. Para esses autores, o 'trabalho em equipe' constitui a menor unidade de produção do cuidado em saúde e caracteriza-se por: intensa interdependência das ações, integração, clareza dos papéis (reconhecimento do papel e trabalho das demais áreas profissionais), compartilhamento de valores, objetivos e identidade de equipe. $\mathrm{O}$ trabalho em equipe se refere a um pequeno grupo de profissionais responsáveis por identificar as necessidades complexas, imprevisíveis e algumas vezes urgentes dos usuários 
e lidar com elas. A 'colaboração' caracteriza-se como forma mais flexível de trabalho interprofissional, com níveis menores de compartilhamento, clareza de papéis e interdependência das ações, e a necessidade de lidar com situações de atendimento um pouco menos imprevisíveis e com menor urgência e complexidade. No 'trabalho em rede', se reconhece maior flexibilidade ainda e menor interdependência das ações, mas mantida a integração em rede. As situações de atendimento são mais previsíveis e menos complexas e urgentes. A rede pode ser virtual no sentido de que os membros podem não interagir pessoalmente e se comunicar de maneira não sincrônica.

Diferente do trabalho em equipe e em rede, nos quais há compartilhamento de identidade e senso de pertencimento (a uma dada equipe ou rede), a colaboração não requer compartilhar identidade ou pertencimento a uma mesma equipe de trabalho, como assinala Reeves e colaboradores (2010, p. 58): "collaboration is a looser form of interprofessional work". D'Amour e colaboradores (2008) chamam de colaboração as situações em que profissionais de diferentes áreas querem trabalhar juntos para prover a melhor atenção à saúde aos usuários, mas ao mesmo tempo reconhecem que têm seus próprios interesses e querem reter algum grau de autonomia. A colaboração pressupõe o desejo de contribuir com o trabalho coletivo e reduzir a competição e as relações de poder entre os profissionais, as quais comprometem a qualidade dos cuidados aos usuários, famílias e comunidade (Peduzzi e Agreli, 2018).

Ainda sobre as diferentes formas de trabalho interprofissional, revisão de literatura examinou os elementos-chave da efetividade da colaboração interprofissional em serviços de atenção primária à saúde com base em estudos que adotaram a observação direta como método principal de pesquisa de campo (Morgan, Pullon e McKinlay, 2015). Os autores destacam a ausência de consenso sobre a concepção de colaboração interprofissional e se esta é equivalente ou não ao trabalho em equipe. Eles propõem o entendimento da colaboração como um termo guarda-chuva que inclui a prática colaborativa interprofissional, denominação utilizada para descrever os elementos da colaboração interprofissional quando presentes na prática dos serviços de saúde, e trabalho em equipe como termo que denota um nível mais profundo de trabalhar juntos com intensa interdependência das ações.

As análises de Morgan, Pullon e McKinlay (2015) e Reeves e colaboradores (2010) sugerem que o trabalho em equipe é a unidade de produção do cuidado à saúde em substituição ao trabalho 'solo' de cada profissional, e é constituído por um pequeno grupo de profissionais responsáveis pela atenção às necessidades de saúde de um conjunto de usuários/pacientes - necessidades essas que podem se apresentar como corriqueiras, complexas, imprevisíveis ou urgentes.

Como apontado, a literatura sobre trabalho em equipe e colaboração interprofissional apresenta atributos ou características que permitem diferenciar 
sua efetividade, isto é, quando uma equipe de saúde é efetiva no cuidado aos usuários e na produção dos melhores resultados. Um dos atributos reconhecidos como condição sine qua non para o trabalho em equipe interprofissional é a interação e comunicação entre os profissionais das diferentes áreas. A revisão realizada por Morgan, Pullon e McKinlay (2015) identificou que o elemento mais importante e tangível da colaboração interprofissional é a oportunidade de comunicação frequente, informal, recíproca e efetiva.

O artigo revisitado (Peduzzi, 2001), apoiado no referencial teórico do processo de trabalho em saúde e na teoria do agir comunicativo, também apresenta critérios de reconhecimento e distinção entre equipes do tipo integração ou agrupamento, que serão descritos a seguir e cotejados com os atributos de trabalho em equipe encontrados na literatura atual sobre o tema.

A comunicação é um componente intrínseco ao trabalho em equipe e à colaboração interprofissional, uma vez que os profissionais de diferentes áreas precisam, em alguma medida, se colocar de acordo quanto ao plano de ação subjacente a executar o cuidado e a atenção à saúde. Os profissionais de diferentes áreas que participam do cuidado de usuários e famílias unem, de forma complementar, ação instrumental e ação comunicativa. $\mathrm{O}$ agir comunicativo implica reconhecer de forma oportuna e pertinente as situações que requerem diálogo, que podem se limitar a uma rápida e informal troca de informação, como apontado (Morgan, Pullon e McKinlay, 2015). Uma das intensas dificuldades do trabalho em equipe reside na crença de que o agir instrumental pode abarcar o conjunto das necessidades de saúde ou mesmo na incapacidade de reconhecer situações que requerem consultar ou escutar os demais envolvidos. Habermas (2012) diagnostica na sociedade contemporânea a colonização da razão instrumental e estratégica em esferas da vida que deveriam ser orientadas pelo agir comunicativo, o que também é observado no trabalho coletivo em serviços de saúde, em particular aqui referido ao trabalho em equipe interprofissional. A literatura sobre trabalho em equipe e colaboração interprofissional destaca como um elemento-chave a comunicação interprofissional.

Outro atributo do trabalho em equipe diz respeito à construção de um projeto assistencial comum. Este se configura como 'comum' por referência aos membros de uma dada equipe, à medida que eles têm sob sua responsabilidade a atenção à saúde dos usuários ou pacientes que atendem. Tomando o processo de trabalho e "suas relações de consubstancialidade com o processo de reprodução das necessidades" (Mendes-Gonçalves, 2017, p. 333), pode-se observar que as ações de saúde executadas em processo - no processo de trabalho - se dão mediante o reconhecimento de necessidades de saúde interpretadas pelos profissionais, à luz de suas concepções de saúde-doença e cuidados (Mendes-Gonçalves, 2017). Portanto, o modo como se organiza o processo de trabalho da equipe e a leitura que os profissionais/equipe fazem 
das necessidades põem em curso ações de saúde conforme um dado modelo de atenção à saúde. Este pode se limitar a reiterar o projeto assistencial biomédico, dominante, ou se constituir como movimento permanente em busca de um olhar e leitura ampliada das necessidades de saúde orientadas pela integralidade da saúde (Ayres, 2004).

A literatura internacional sobre trabalho em equipe destaca a definição de objetivos comuns compartilhados entre os membros da equipe como importante preditor de sua efetividade, embora os próprios autores refiram que seus estudos mostram que é comum que os integrantes das equipes não tenham clareza sobre quais são exatamente seus objetivos no cuidado dos pacientes sob sua responsabilidade (West e Lyubovnikova, 2013). A elaboração de objetivos comuns (Reeves et al., 2010; Reeves, Xyrichis e Zwarenstein, 2018; D’Amour et al., 2008) requer que a equipe conheça as características e as necessidades de saúde dos usuários/pacientes e respectivas famílias e, a partir destes e do contexto de trabalho em que atua, defina prioridades de cuidado.

O trabalho em equipe constitui uma das configurações contemporâneas da divisão social do trabalho em saúde. Mendes-Gonçalves (2017) apresenta análise criteriosa do processo de divisão de trabalho que se dá no campo da saúde a partir da ressignificação do hospital como espaço de tratamento e cura, na passagem do século XVIII para o XIX (Foucault, 2002). A divisão social do trabalho constitui a expressão da cisão entre as partes 'mais intelectuais' e 'mais manuais', visto que todo trabalho retém componente intelectual, e também expressão da divisão de classe e segmentos sociais, à medida que

a técnica não existe isolada de sua apropriação diferenciada na reprodução das diferenças de classe, e o controle dos momentos 'mais intelectuais' do trabalho garante o poder sobre o conjunto do processo, além de outras hierarquizações sociais importantes (Mendes-Gonçalves, 2017, p. 356, destaque no original).

Esse processo constitui relações hierárquicas entre as diferentes áreas profissionais que se transmutam em trabalhos com desigual valor social - o que acarreta, efetivamente, relações de trabalho nas quais alguns trabalhadores são considerados superiores a outros, todos executando ações que constituem, no seu conjunto articulado, atenção à saúde (Peduzzi, 2001).

A divisão social do trabalho que transmuta diferenças técnicas em desigual valor social dos agentes, incluindo remuneração e condições de trabalho como a carga horária semanal, também está presente em outro critério que permite diferenciar as equipes integração e agrupamento: especificidades dos trabalhos especializados versus flexibilidade da divisão do trabalho, que se refere à tensão entre, de um lado, a tendência crescente de especialização e a consequente fragmentação da atenção à saúde, e de outro a imediata e recíproca necessidade de articulação e integração das ações e dos agentes. 
A autonomia técnica ou autonomia profissional também é expressão do processo de divisão do trabalho e está em diferentes graus presente em todos os trabalhos especializados ou profissões da saúde, visto que no campo da saúde não é possível a total e prévia padronização de todas as ações de cuidado. Espera-se que as necessidades de saúde sejam reconhecidas, interpretadas e negociadas entre profissionais e usuários/pacientes de forma ampliada e contextualizada, em cada encontro, daí a impossibilidade da total estandardização das ações de cuidado e a necessidade de algum grau de liberdade na tomada de decisão técnica. Porém, o trabalho em equipe requer que cada profissional abra mão da expectativa de plena autonomia pautada no saber técnico científico de sua área de atuação e reconheça a esfera de autonomia dos demais, ou seja, que reconheça o trabalho dos demais profissionais da equipe e a interdependência entre eles. $\mathrm{O}$ reconhecimento do trabalho dos demais e sua interdependência permite a articulação das ações executadas por profissionais de diferentes áreas e é apontado na literatura sobre o tema como atributo do trabalho em equipe e colaboração interprofissional (Canadian Interprofessional Health Collaborative, 2010; Reeves et al., 2010).

Nesse sentido, retoma-se a ideia de que a concepção de colaboração interprofissional, correlata ao trabalho em equipe, se refere às situações de trabalho nas quais os profissionais de saúde querem trabalhar juntos porque reconhecem que juntos produzirão melhores resultados no cuidado e atenção à saúde de usuários, famílias e comunidade ( $\mathrm{D}^{\prime}$ Amour et al., 2008). Contudo, querem também, em alguma medida, preservar seus próprios interesses e algum grau de autonomia. Cabe destacar que a cooperação decorrente da divisão social do trabalho se refere ao "agrupamento das capacidades individuais potencializadas pelo encontro com outras, forjando uma capacidade coletiva superior" (Soares, Souza e Campos, 2016, p. 46).

Um estudo aponta forte relação entre colaboração e atenção centrada no usuário, as quais, juntas, constituem movimento das equipes em incluir os usuários, suas famílias e comunidade como protagonistas e partícipes do 'fazer junto' na equipe interprofissional (Agreli, Peduzzi e Silva, 2016). A atenção centrada no usuário reitera e destaca a necessidade de mediações reflexivas e dialógicas dos profissionais das equipes com usuários, famílias e comunidade, na construção de espaços de entendimento que precisam ser fortalecidos e ampliados - do âmbito da definição de planos de cuidados à esfera pública pautados no reconhecimento da saúde como valor e direito.

Pautado, sobretudo, nas considerações teóricas do trabalho interprofissional de Peduzzi (2001), estudo empírico desenvolvido no contexto da atenção primária do SUS evidencia que a colaboração se caracteriza como uma forma de trabalho essencial para a configuração do trabalho coletivo em saúde orientado às necessidades de saúde de usuários, famílias e comunidade e pode ocorrer de formas distintas e complementares (Agreli, 2017; Agreli, Peduzzi e Bailey, 
2017a): colaboração no âmbito das equipes, quando os profissionais buscam alternativas entre os próprios membros da equipe para melhorar a qualidade da atenção à saúde; colaboração em rede e com usuários e comunidade, quando os profissionais da equipe buscam alternativas na equipe e também em outros serviços, setores e com os usuários, família e comunidade. Essa modalidade de colaboração ressalta a importância do trabalho interprofissional em equipe na promoção do trabalho intersetorial e da participação social (Figura 1).

\section{Figura 1}

\section{Diferentes formas de trabalho interprofissional}

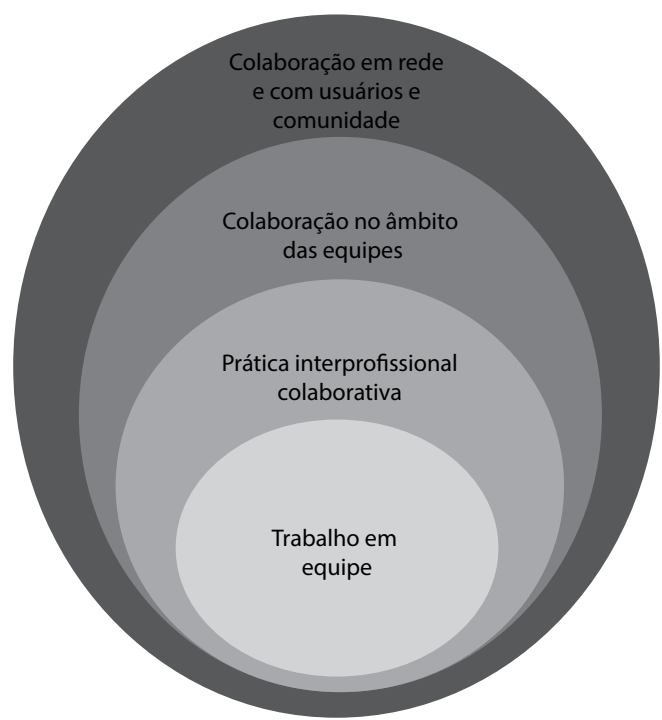

Fonte: Adaptação e tradução de Morgan, Pullon e McKinlay (2015) e Reeves e colaboradores (2010), adaptação de Agreli (2017) e Peduzzi e Agreli (2018).

Outro construto capaz de facilitar a compreensão e operacionalização da colaboração interprofissional é o de clima do trabalho em equipe (Agreli, 2017; Agreli, Peduzzi e Bailey, 2017a). O clima pode ser entendido como percepção individual ou como percepção compartilhada em pequenos grupos de pessoas ou mesmo da organização (Schneider, Ehrhart e Macey, 2013). Para Anderson e West (1998), o clima do trabalho em equipe é definido como o conjunto de percepções e significados compartilhados entre os membros de uma equipe acerca de políticas, práticas e procedimentos que eles vivenciam no trabalho. Segundo esses autores, o clima de equipe se expressa em quatro dimensões: participação segura, que diz respeito ao âmbito das interações e comunicação entre os membros da equipe, no sentido de sentirem segurança e confiança para opinar sem receio de cerceamentos dos colegas; apoio para novas ideias, existência de suporte concreto, prático, para que cada integrante ou a equipe 
apresente novas formas de responder a situações ou necessidades do cotidiano de trabalho; objetivos da equipe, existência de objetivos claros e compartilhados entre os integrantes da equipe; orientação para a tarefa, existência de compromisso individual e da equipe de acompanhamento, monitoramento e análise crítica do desempenho, tendo em vista alcançar os objetivos traçados com a melhor qualidade possível (Silva et al., 2016).

Colaboração e clima do trabalho em equipe possuem elementos conceituais comuns que sugerem que a compreensão de aspectos macrossociais da organização do trabalho interprofissional, como a colaboração para estabelecimento da Rede de Atenção à Saúde (RAS), inclui o estudo de aspectos do âmbito microssocial (da interação social) no contexto imediato do trabalho em equipe (Peduzzi e Agreli, 2018).

Para Agreli, Peduzzi e Bailey (2017b), as áreas de interseção entre colaboração e clima de equipe são descritas como: interação e comunicação entre membros da equipe; objetivos comuns em torno dos quais o trabalho coletivo é organizado; responsabilidade compartilhada pela orientação do trabalho para a excelência; e promoção da inovação no trabalho. A compreensão de tais aspectos se faz necessária para avanço das proposições do trabalho coletivo tanto no micro quanto no macrocontexto da organização do trabalho interprofissional.

\section{Considerações finais}

A tendência crescente de trabalho interprofissional relacionada às mudanças do perfil demográfico e epidemiológico da população brasileira e mundial, assim como ao reconhecimento da complexidade das necessidades de saúde e da rede de serviços e do sistema de saúde (SUS), motivou a revisita ao conceito de trabalho em equipe, sua tipologia e atributos, em diálogo com a literatura internacional sobre o tema.

A revisita mostrou atualidade da abordagem do trabalho em equipe no contexto do SUS, na perspectiva teórica do processo de trabalho em saúde e das racionalidades instrumental e comunicativa, cujos atributos se aproximam dos apontados na literatura e dialogam com eles.

Trabalho em equipe interprofissional é entendido como uma forma de trabalho coletivo que se configura na relação recíproca entre as intervenções técnicas e as interações dos múltiplos agentes envolvidos, visto que requer, de um lado, a articulação das ações das diversas áreas profissionais, a partir do reconhecimento da sua interdependência, e de outro a complementaridade entre agir instrumental e agir comunicativo. A discussão com a literatura mostra que o trabalho em equipe também constitui uma das formas de trabalho interprofissional, com a colaboração interprofissional e a prática colaborativa, e que a colaboração pode se dar na equipe ou no trabalho em rede com usuários e comunidade. Os atributos ou características do trabalho em 
equipe são: comunicação interprofissional, objetivos comuns, reconhecimento do trabalho dos demais componentes da equipe, interdependência das ações, colaboração interprofissional e atenção centrada no usuário.

Destaca-se a potencialidade de mudança que reside no trabalho em equipe efetivo e sua contribuição para a qualidade da atenção à saúde e produção de saúde. $\mathrm{O}$ reconhecimento da circularidade entre processo de trabalho e necessidades de saúde possibilita às equipes novas leituras, mais amplas e abrangentes das necessidades, que por sua vez se desdobram na organização do trabalho interprofissional pautado na prática comunicativa intersubjetiva entre os trabalhadores das equipes e destes com os usuários, famílias e população.

Por fim, verifica-se a necessidade de se aumentarem as investigações sobre o tema no cenário do SUS, tanto na atenção primária como na especializada. Destaca-se a utilização de método mistos que permitem tanto pesquisas conceituais e de experiências em curso, quanto a validação e aplicação de instrumentos de medida e avaliação da efetividade do trabalho em equipe e prática colaborativa. De igual importância é a ampliação do debate e implementação da educação interprofissional no país, imprescindível para o fortalecimento do trabalho em equipe integrado e a formação de profissionais de saúde com competências colaborativas.

\section{Financiamento}

Este suplemento “Educação e Trabalho em Saúde: diálogos e experiências no Brasil e em Portugal" foi realizado com apoio da Coordenação de Aperfeiçoamento de Pessoal de Nível Superior (Capes) - Código de Financiamento 001; e do Departamento de Pesquisa em História das Ciências e da Saúde da Casa de Oswaldo Cruz (Depes/COC/Fiocruz, 6151000000). Especificamente este artigo contou com auxílio FAPESP PPSUS-SP 2016-2018 (processo FAPESP: 2016/14975-1).

Todos os autores declaram que não há conflito de interesses.

\section{Agradecimentos}

Agradecemos ao professor Scott Reeves, da Kingston University \& St. George's University of London (in memoriam), pelas suas contribuições ao grupo de pesquisa Gestão e Educação em Saúde e Enfermagem na Perspectiva Colaborativa Interprofissional. 


\section{TRABAJO DE EQUIPO: REVISITANDO EL CONCEPTO Y SUS}

\section{CONSECUENCIAS PARA EL TRABAJO INTERPROFESIONAL}

Resumen La creciente complejidad de las necesidades de salud que requieren un enfoque amplio y contextualizado y de la organización de los servicios en red, ha motivado la revisita al concepto de trabajo en equipo, a la tipología equipo-integración y agrupación, y a sus atributos. El efectivo trabajo en equipo se constituye como expresión, por un lado, de la articulación de las acciones de las diversas áreas a partir del reconocimiento de su interdependencia y, de otro lado, de la complementación indispensable entre actuar instrumentalmente y actuar comunicativamente. Cabe señalar que el trabajo en equipo es también una expresión de la división social del trabajo y la transmutación de diferencias técnicas en valor social desigual del trabajo especializado de las distintas profesiones y, por lo tanto, de sus agentes. Las contradicciones presentes en los procesos de trabajo, en su consubstancialidad a las necesidades de salud, pueden limitarse a reiterar los modelos dominantes de atención a la salud o buscar su superación por mediaciones reflexivas y dialógicas de los trabajadores de los equipos entre sí y de estos con usuarios, familias y población. Finalmente, el trabajo en equipo constituye una de las formas de trabajo interprofesional junto con la colaboración interprofesional y la práctica colaborativa, y esta colaboración puede tener lugar en equipo o en red con los usuarios y la comunidad.

Palabras clave equipo de asistencia al paciente; relaciones interprofesionales; trabajo.

\section{Nota}

1"Tal como os indivíduos exteriorizam sua vida, assim são eles. O que eles são coincide, pois, com sua produção, 'tanto com o que produzem como também com o modo como produzem'. O que os indivíduos são, portanto, depende das condições materiais de sua produção" (Marx e Engels, 2007, p. 87, destaque nosso). 


\section{Referências}

AGRELI, Heloise L. F. Prática interprofissional colaborativa e clima do trabalho em equipe na APS. 262 fl. Tese. (Doutorado em Fundamentos e Práticas em Gerenciamento em Enfermagem) - Escola de Enfermagem, Universidade de São Paulo, São Paulo, 2017.

AGRELI, Heloise F.; PEDUZZI, Marina; BAILEY, Christopher. The relationship between team climate and interprofessional collaboration: preliminary results of a mixed methods study. Journal of Interprofessional Care, London, v. 31, n. 2, p. 184-186, 2017 a.

AGRELI, Heloise F.; PEDUZZI, Marina; BAILEY, Christopher. Contributions of team climate in the study of interprofessional collaboration: a conceptual analysis. Journal of Interprofessional Care, London, v. 31 , n. 6 , p. $679-684,2017$ b.

AGRELI, Heloise F.; PEDUZZI, Marina; SILVA, Mariana C. Atenção centrada no paciente na prática interprofissional colaborativa. Interface: Comunicação, Saúde, Educação, Botucatu, v. 20, n. 59, p. 905-916, 2016

ANDERSON, Neil R.; WEST, Michael A. Measuring climate for work group innovation: development and validation of team climate inventory. Journal of Organizational Behavior, Oxford, v. 19, n. 3, p. 235-258, 1998. Disponível em: <http://www.psychwiki.com/dms/other/ labgroup/Measufsdfsdbger345resWeekl/Danika/ Andersen1998.pdf>. Acesso em: 20-08-2018.

AROUCA, Sergio. O dilema preventivista: contribuição para a compreensão e crítica da medicina preventiva. Rio de Janeiro: Editora Fiocruz/Unesp, 2003.

ARTMANN, Elizabeth. Interdisciplinaridade no enfoque intersubjetivo habermasiano: reflexões sobre planejamento e Aids. Ciência \& Saúde Coletiva, Rio de Janeiro, v. 6, n. 1, p. 183-195, 2001. Disponível em: <http://www. scielo.br/scielo.php?script=sci_arttext\&pi$\mathrm{d}=\mathrm{S} 1413=81232001000100015-\& \operatorname{lng}=\mathrm{en} \& \mathrm{nrm}$ iso >. Acesso em: 20-08-2018.
AYRES, José R. C. M. Sujeito, intersubjetividade e práticas de saúde. Ciência \& Saúde Coletiva, Rio de Janeiro, v. 6, n. 1, p. 63-72, 2001.

AYRES, José R. C. M. O cuidado, os modos de ser (do) humano e as práticas de saúde. Saúde e Sociedade, São Paulo, v. 13, n. 3, p. 16-29, 2004. Disponível em: <http://www. scielo.br/scielo.php?script $=$ sci_arttext \&pi$\mathrm{d}=$ S0104=12902004000300003-\&lng =en \&nrmiso >. Acesso em: 19-08-2018.

BRANDT, Barbara et al. A scoping review of interprofessional collaborative practice and education using the lens of Triple Aim. Journal of Interprofessional Care, London, v. 28, n. 5, p. 393-399, 2014.

CANADIAN INTERPROFESSIONAL HEALTH COLLABORATIVE (CIHC). A national interprofessional competency framework. University of British Columbia, Canada, Vancouver, 2010. Disponível em: <https://www.cihc.ca/files/ CIHC_IPCompetencies_Feb1210.pdf $>$ Acesso em: 20-08-2018.

CHANG, Wen-Yin et al. Job satisfaction and perceptions of quality of patient care, collaboration and teamwork in acute care hospitals. Journal of Advanced Nursing, New York, v. 65, n. 9, p. 1.946-1.955, 2009.

CHIANG VEGA, María M. et al. Clima organizacional y satisfacción laboral: una comparación entre hospitales públicos de alta y baja complejidad. Salud de Trabajadores, Linares Alcantara, v. 19, n. 1, p. 5-16, 2011.

D'AMOUR, Danielle et al. A model and typology of collaboration between professionals in healthcare organizations. BMC Health Services Research, London, v. 8, n. 1, p. 1-14, 2008.

DONNANGELO, Maria C.; PEREIRA, Luiz. Saúde e sociedade. São Paulo: Livraria Duas Cidades, 1976. 
FOUCAULT, Michel. O nascimento da clínica. Rio de Janeiro: Forense Universitária, 2002.

FURTADO, Juarez P. Equipes de referências: arranjo institucional para potencializar a colaboração entre as disciplinas e profissões. Interface: Comunicação, Saúde, Educação, Botucatu, v. 11, n. 22, p. 239-255, 2007.

HABERMAS, Jürgen. Consciência moral e agir comunicativo. 2. ed. Rio de Janeiro: Tempo Brasileiro, 2003a.

HABERMAS, Jürgen. Mudança estrutural da esfera pública: investigações quanto a uma categoria da sociedade burguesa. 2. ed. Rio de Janeiro: Tempo Brasileiro, 2003b.

HABERMAS, Jürgen. Técnica e ciência como ideologia. Lisboa: Edições 70, 2009.

HABERMAS, Jürgen. Teoria do agir comunicativo I: racionalidade da ação e racionalização social. São Paulo: Martins Fontes, 2012.

HOLLENBECK, John; BEERSMA, Bianca; SCHOUTEN, Maartje. Beyond team types and taxonomies: a dimensional scaling conceptualization for team description. Academy of Management Review. New York, v. 37, n. 1, p. 82-106, jan. 2012.

MARX, Karl; ENGELS, Friedrich. A ideologia alemã: crítica da mais recente filosofia alemã em seus representantes Feuerbach, B. Bauer e Stirner, e do socialismo alemão em seus diferentes profetas (1845-1846). São Paulo: Boitempo, 2007.

MENDES-GONÇALVES, Ricardo B. Tecnologia e organização social das práticas de saúde: características tecnológicas do processo de trabalho na rede estadual de centros de saúde de São Paulo. São Paulo: Hucitec, 1994.

MENDES-GONÇALVES, Ricardo B. Prática de saúde: processo de trabalho e necessidades. In: AYRES, José R. C. M.; SANTOS, Liliana (orgs.). Ricardo Bruno Mendes-Gonçalves: saúde, sociedade e história. São Paulo: Hucitec; Porto
Alegre: Editora da Rede Unida, 2017. p. 298374. (Saúde em Debate).

MORGAN, Sonia; PULLON, Susan; McKINLAY, Eileen. Observation of interprofessional collaborative practice in primary care teams: an integrative literature review. International Journal of Nursing Studies, London, v. 52, n. 7, p. 1.217-1.230, 2015. Disponível em: <https:// www.ncbi.nlm.nih.gov/pubmed/25862411>. Acesso em: 20-08-2018.

ORGANIZAÇÃO MUNDIAL DA SAÚDE (OMS). Marco para ação interprofissional e prática colaborativa. Rede de Profissionais da Saúde, Enfermagem e Obstetrícia. Recursos Humanos em Saúde. Genebra, OMS 2010. Disponível em: <http://www.paho.org/bra/images/stories/documentos/marco_para_acao.pdf\%20>. Acesso em 20-08-2018.

PEDUZZI, Marina. Equipe multiprofissional de saúde: conceito e tipologia. Revista de Saúde Pública, São Paulo, v. 35, n. 1, p. 103-109, 2001.

PEDUZZI, Marina. Trabalho em Equipe de saúde da perspectiva de gerentes de serviços de saúde: possibilidades da prática comunicativa orientada pelas necessidades de saúde dos usuários e da população. 247 fl. Tese (Livre Docência em Gestão de Recursos Humanos em Saúde e Enfermagem) - Escola de Enfermagem, Universidade de São Paulo, São Paulo, 2007.

PEDUZZI, Marina. Trabalho em equipe. In: PEREIRA, Isabel B.; LIMA, Júlio C. F. (orgs.). Dicionário de educação profissional em saúde. 2 ed. rev. ampl. Rio de Janeiro: EPSJV/Fiocruz, 2009. p. 419-426.

PEDUZZI, Marina; AGRELI, Heloise F. Trabalho em equipe e prática colaborativa na atenção primária à saúde. Interface: Comunicação, Saúde, Educação, Botucatu, v. 2, supl. 22, p. 1.525-1.534, 2018.

PEDUZZI, Marina et al. Educação interprofissional: formação de profissionais de saúde para o trabalho em equipe com foco nos usuários. Revista Escola de Enfermagem da USP, São Paulo, v. 47, n. 4, p. 977-983, 2013. Disponível 
em: <http://www.scielo.br/scielo.php?scrip$\mathrm{t}=$ sci_arttext\&pid=S0080-62342013000400977> . Acesso em: 20-08-2018.

REEVES, Scott et al. Interprofessional teamwork for health and social care. Oxford: Wiley-Blackwell, 2010.

REEVES, Scott et al. A BEME systematic review of the effects of interprofessional education: BEME Guide n. 39. Medical Teacher, Dundee, v. 38, n. 7, p. 656-668, 2016. Disponível em: <https:// www.ncbi.nlm.nih.gov/pubmed/27146438>. Acesso em: 20-08-2018.

REEVES, Scott et al. Interprofessional collaboration to improve professional practice and healthcare outcomes. Cochrane Database Systematic Reviews, v. 6, n. CD000072, 2017.Disponível em: <https://www.ncbi.nlm.nih.gov/ pubmed/28639262>. Acesso em: 20-08-2018.

REEVES, Scott; XYRICHIS, Andreas; ZWARENSTEIN, Merrick. Teamwork, collaboration, coordination, and networking: why we need to distinguish between different types of interprofessional practice. Journal of Interprofessional Care, London, v. 32, n. 1, p. 1-3, 2018.

RIVERA, Francisco J. U. Análise estratégica em saúde e gestão pela escuta. Rio de Janeiro: Editora Fiocruz, 2003.

RIVERA, Francisco J. U.; ARTMANN, Elizabeth. Planejamento e gestão em saúde: flexibilidade metodológica e agir comunicativo. Ciência \& Saúde Coletiva, Rio de Janeiro, v. 4, n. 2, p. 355-365, 1999.

SCHNEIDER, Benjamin; EHRHART, Mark G.; MACEY, William H. Organizational climate and culture. Annual Review of Psychology, Palo Alto, v. 64, p. 361-388, 2013.

SCHRAIBER, Lilia B. et al. Planejamento, gestão e avaliação em saúde: identificando problemas. Ciência \& Saúde Coletiva, Rio de Janeiro, v. 4, n. 2, p. 221-242, 1999.
SILVA, Mariana C. et al. Cross-cultural adaptation and validation of the teamwork climate scale. Revista de Saúde Pública, São Paulo, v. 50, n. 52, 2016. Disponível em: <http://www. scielo.br/scielo.php?script $=$ sci_arttext\&pi$\mathrm{d}=$ S0034=89102016000100237-\&lng=en\&nrmiso >. Acesso em: 19-08-2018.

SOARES, Cassia B.; SOUZA, Helton S.; CAMPOS, Celia M. S. Processo de trabalho e enfermagem: uma contribuição a partir da saúde coletiva. In: SOUZA, Helton S.; MENDES, Áquilas (orgs). Trabalho \& saúde no capitalismo contemporâneo: enfermagem em foco. Rio de Janeiro: DOC Saberes, 2016. p. 43-61.

SONG, Hummy et al. Team dynamics, clinical work satisfaction, and patient care coordination between primary care providers: a mixed methods study. Health Care Management Review, Charlotte, v. 42, n. 1, p. 28-41, 2017.

WEST, Michael A.; LYUBOVNIKOVA, Joanne. Illusions of team working in health care. Journal of Health Organization and Management, Bingley, v. 27, n. 1, p. 134-142, 2013.

WEST, Michael; RICHTER, Andreas. W. Team climate and effectiveness outcomes. In: ASHKANASY, Neal M.; WILDEROM, Celeste P.; PETERSON, Mark F. (eds.). Handbook of organizational culture and climate. Thousand Oaks, Sage, 2011. p. 249-270.

XYRICHIS, Andreas; REEVES, Scott; ZWARENSTEIN, Merrick. Examining the nature of interprofessional practice: an initial framework validation and creation of the InterProfessional Activity Classification Tool (InterPACT). Journal of Interprofessional Care, London, v. 32, n. 44, p. 416-425, 2018.

ZWARENSTEIN, Merrick; GOLDMAN, Joanne; REEVES, Scott. Interprofessional collaboration: effects of practice-based interventions on professional practice and healthcare outcomes. Cochrane Database Systematic Reviews, s.l., v. 3, n. CD000072, 2009. 